\title{
Psychological effects of dopamine agonist treatment in patients with hyperprolactinemia and prolactin-secreting adenomas
}

\author{
Adriana G Ioachimescu' , Maria Fleseriu², Andrew R Hoffman ${ }^{3,5}$, T Brooks Vaughan III ${ }^{4}$ and Laurence Katznelson ${ }^{3}$ \\ ${ }^{1}$ Division of Endocrinology, Diabetes and Metabolism, Departments of Medicine, Neurosurgery and Emory Pituitary Center, Emory \\ University School of Medicine, Atlanta, Georgia, USA, ${ }^{2}$ Division of Endocrinology, Diabetes and Clinical Nutrition, \\ Departments of Medicine, Neurosurgery and Northwest Pituitary Center, Oregon Health and Science University, \\ Portland, Oregon, USA, ${ }^{3}$ Division of Endocrinology, Gerontology and Metabolism, Department of Medicine and \\ Neurosurgery, Stanford University School of Medicine, Stanford, California, USA, ${ }^{4}$ Division of Diabetes, Endocrinology \\ and Metabolism and Veteran Affairs Hospital, Department of Medicine and Neurosurgery, University of Alabama at \\ Birmingham, Birmingham, Alabama, USA, and ${ }^{5}$ Veteran Affairs, Palo Alto, California, USA \\ Correspondence \\ should be addressed \\ to A G loachimescu \\ Email \\ aioachi@emory.edu
}

\begin{abstract}
Background: Dopamine agonists (DAs) are the main treatment for patients with hyperprolactinemia and prolactinomas. Recently, an increasing number of reports emphasized DAs' psychological side effects, either de novo or as exacerbations of prior psychiatric disease.

Methods: Review of prospective and retrospective studies (PubMed 1976, September 2018) evaluating the psychological profile of DA-treated patients with hyperprolactinemia and prolactinomas. Case series and case reports of psychiatric complications were also reviewed.

Results: Most studies were cross-sectional and had a control group of healthy volunteers or patients with nonfunctioning pituitary adenomas. There were few prospective studies, with/without control group, that included small numbers of patients. Compared with controls, patients with hyperprolactinemia generally had worse quality of life, anxiety, depression and certain personality traits. Patients receiving DAs had higher impulsivity scores than normoprolactinemic controls. Impulse control disorders (ICDs) were reported in both genders, with hypersexuality mostly in men. Multiple ICDs were sometimes reported in the same patient, usually reversible after DA discontinuation. In case reports, DA therapy was temporally associated with severe depression, manic episodes or psychosis, which improved after discontinuation and administration of psychiatric medications. Gender type of DA, dose and duration of therapy did not correlate with occurrence of psychiatric pathology.

Conclusion: Patients with hyperprolactinemia receiving DAs may develop changes in mood and behavior regardless of prior psychiatric history. Increased awareness for ICDs, depression, mania and other types of psychosis is needed by all physicians who prescribe DAs. Larger prospective controlled clinical studies are needed to delineate prevalence, risk stratification and management.
\end{abstract}

\section{Introduction}

Dopamine agonists (DAs) constitute the first-line treatment for prolactin-secreting adenomas (prolactinomas), the most common type of secretory pituitary adenoma.
Treatment is recommended to restore gonadal function, decrease galactorrhea, lower prolactin levels and decrease tumor size $(1,2)$. Treatment is long term in most patients (3). 
Bromocriptine and cabergoline are currently approved for this indication in the United States; bromocriptine is also approved for treatment of type 2 diabetes. Quinagolide is another DA currently or previously used in some countries other than the United States.

The most frequent adverse effects of DAs are nausea, dizziness, orthostatic hypotension and headaches. In addition, a variety of psychological side effects have also been described, many as isolated case reports. Psychosis, mania, anxiety, depression, confusion, auditory hallucinations, hyperactivity, insomnia, nightmares, paranoia and impulse control disorders (ICDs) have been reported either de novo after starting DA therapy or as exacerbations of previously known psychiatric disease. Many endocrinologists are not aware of the association between DAs and psychiatric symptoms, and as a result, underreporting is likely. Moreover, there are no specific guidelines for the management of patients experiencing psychological problems as a result of DA therapy.

In this review, we examine the current knowledge regarding mechanisms, clinical presentation, course and management of psychological disturbances encountered in patients treated with DAs for hyperprolactinemia.

\section{Methods}

A US National Library of Medicine PubMed search through July 2018 was conducted using the search terms 'dopamine agonists' or 'bromocriptine' or 'cabergoline' and 'psychiatric disorders', 'impulse control disorders' or 'hypersexuality' 'depression' or 'mania'. All English language articles were read and reviewed by the authors for relevance and significance.

\section{Mechanism of psychiatric disturbances in patients with prolactinomas treated with dopamine agonists}

The endocrine system and mental health are closely entwined, especially when the hypothalamus and pituitary gland are involved. Dopamine is a neurotransmitter important for motor function, motivation, reinforcement learning and prolactin control. There are three main dopaminergic pathways: (1) nigrostriatal, involved in motor functions, (2) mesocorticolimbic, also known as the reward system, involved in the regulation of behavior, pleasure and addiction and (3) tuberoinfundibular, involved in the control of prolactin secretion (4).
DAs exert their endocrine effects by binding to dopamine type 2 (D2) receptors in the tuberoinfundibular system (5). Both D2 and D3 receptors are also expressed in the striatal pathway (6), and D3 receptors are present in high density in the mesolimbic cortex. DA binding is generally not specific to one receptor type from a certain neuronal pathway, which can explain some of the neuropsychiatric side effects of DAs (7). Bromocriptine, cabergoline and quinagolide bind with high affinity to D2 and with lower affinity to D1 and D3 receptors. Quinagolide and cabergoline have lower affinity for D1 than bromocriptine (8). Pramipexole and ropinirole, DAs approved for Parkinson's disease (PD) and restless leg syndrome (RLS), have a higher affinity for D3 compared with bromocriptine and cabergoline (9). Notably, selective D3 agonists were associated with more ICDs (10) in patients receiving DAs for PD and RLS.

Central nervous system (CNS) side effects depend on the drug's capacity to cross the blood-brain barrier, which is controlled by transporter molecules. P-glycoprotein 1 (P-gp) is a transport molecule encoded by the $A B C B 1$ gene that transports substrates released from the neuron back into the blood. Genetic polymorphisms of the $A B C B 1$ gene may influence or alter the function of the P-gp protein and could influence the predisposition to CNS side effects from DA therapy. This hypothesis is supported by an experimental ABCB1-knockout mouse model study and a case-control study of 79 patients with prolactinomas. In the latter, patients completed questionnaires to determine whether fatigue, dizziness, sleep disorders and headaches changed during cabergoline therapy. ABCB1 SNPs rs1045642 were associated with fatigue and sleep disorders, while SNP rs2032582 associated with dizziness during DA therapy (11).

Whether patients with hyperprolactinemia are more susceptible to neuropsychiatric complications of DAs than individuals with normal prolactin levels remains to be determined. Several factors have been invoked, including central dopamine depletion (5), decreased gonadal sex steroid levels or hyperprolactinemia itself. It is difficult to tease them apart as they often occur simultaneously. Prolactin levels can increase during stress, and studies in normal subjects have reported surges in serum prolactin in induced situations that evoked feelings of rage and humiliation (12).

In summary, the mechanisms of psychologic and psychiatric disturbances in patients with prolactinomas treated with DAs are complex and incompletely understood. They entail specific affinities of the drugs on different classes of DA receptors in the brain, as well as the altered baseline tone of dopaminergic activity. 


\section{Psychological profile of patients with hyperprolactinemia}

Early reports in the 1970-1990s included 3 to 14 patients and indicated increased levels of anxiety, distress and hostility $(13,14,15,16)$ and that treatment with bromocriptine was associated with amelioration of these symptoms $(15,16,17)$. One study had a doubleblind cross-over placebo-controlled design and included eight patients with hyperprolactinemia (16). However, a prospective study in nine patients treated with bromocriptine for 6-12 months (without a control group) did not reproduce these findings (18). In a cross-sectional study of ten women with hyperprolactinemia, patients were more likely to experience depression, anxiety and hostility than normoprolactinemic controls with amenorrhea (17).

A study in 39 patients with untreated prolactinoma had lower scores on the SF-36 questionnaire (36-Item Short Form Survey, a patient reported survey of health) compared with healthy controls. The domains affected were role physical, vitality, social functioning, role emotional, mental health and the mental summary. The physical functioning, bodily pain, general health and physical summary were similar to controls (19).

There are few controlled clinical studies that report systematic evaluation of neurocognitive and psychological functions in hyperprolactinemic patients at baseline and during DA treatment. In a single-center cross-sectional study of women treated with bromocriptine, cabergoline or quinagolide for microprolactinomas, 81 patients were invited to complete questionnaires (62 accepted) and were asked to provide a control person of comparable age and same sex (a relative, friend or neighbor). The control group consisted of 183 subjects. The healthrelated questionnaires (Nottingham Health Profile-NHP, SF-36, Multidimensional Fatigue Inventory-MFI-20 and Hospital Anxiety and Depression Scale-HADS) covered different dimensions of quality of life, including physical, emotional, mental and social aspects. The subscale for social functioning and role limitations due to physical problems on SF-36, the energy, emotional reaction and social isolation subscales on NHP, and general and physical, fatigue, activity and motivation subscales on MFI-20 were all impaired in the hyperprolactinemic patients when compared with controls. Furthermore, prolactinoma patients also had worse anxiety, depression and total scores for HADS compared with controls. Of note, the extent of psychological impairment did not correlate with either current DA use or duration of therapy. In addition, neither the serum prolactin level, nor the presence of hypogonadism in premenopausal women correlated with the degree of quality of life and psychological impairments (20).

A study in 86 patients with prolactinomas described a distinct dopaminergic personality profile using the Eysenck Personality questionnaire and the Cloninger Temperament and Personality Questionnaire. Patients with prolactinoma were compared with 58 patients with nonfunctioning pituitary adenomas (NFA) and 172 controls. All patients with pituitary tumors had increased neuroticism, increased fear or uncertainty and increased fatigability and asthenia compared with controls. Patients with prolactinoma had higher neuroticism, reduced extraversion, increased shyness with strangers and lower scores on novelty seeking subscale impulsiveness compared with patients with NFA. A subgroup analysis in 55 patients receiving DAs showed that these patients were more dependent than 31 non-treated patients. In addition, treated patients who achieved normal serum prolactin levels had lower novelty seeking, exploratory excitability and extravagance tendencies compared with those with persistent hyperprolactinemia (21). This study supports the theory that patients with hyperprolactinemia have low dopaminergic activity, which has been associated with decreased novelty seeking and increased harm avoidance scores. Interestingly, these personality traits did not change after DA therapy in this study.

A recent study evaluated 25 patients with prolactinoma and 32 healthy controls with the revised Minnesota Impulsive Disorders Interview, Barratt Impulsiveness Scale, Symptom Check List questionnaire, Beck Depression Inventory and Beck Anxiety Inventory. At baseline, there were no differences between groups regarding somatization, anxiety, obsession, depression, interpersonal sensitivity, psychoticism, hostility, phobic anxiety or paranoid ideation. Similarly, there were no inter-group differences after 3 months of cabergoline treatment. More patients in the prolactinoma group screened positive for obsession, interpersonal sensitivity and paranoid ideation after 12 months of treatment (22).

In summary, based on available literature, patients with hyperprolactinemia appear to have decreased quality of life, and different personality profiles compared with control subjects. Some, but not all studies also indicate increased anxiety and depression. It is not clear whether DA therapy favorably influences the selfreported mental and physical health of patients with hyperprolactinemia. Further large controlled studies are needed to clarify this issue. 


\section{Dopamine agonist therapy and impulse control disorders}

The ICDs are 'disruptive, impulse control and conduct disorders' defined as the failure to resist an impulse or the temptation to perform an act that is harmful to oneself or others. Manifestations include pathological gambling, hypersexuality, binge eating, compulsive shopping, compulsive medication use, obsessive hobbying and punding. Punding is defined as compulsive repetitive mechanical tasks (i.e. assembling and disassembling or sorting objects). ICDs can be serious with significant social and financial consequences.

The prevalence of ICD in the general population is estimated at $8 \%$ (7). A retrospective study based on FDA Adverse Event Reporting System between 2003 and 2012 identified 1580 ICD events. Of them, DA therapy accounted for 710 events (45\%): pramipexole (410), ropirinole (188), cabergoline (56) and bromocriptine (30). The study indicated affected patients had a median age of 55 and male gender preponderance (65.8\%). There was a slightly higher reporting by patients than physicians. The FDA reporting increased progressively after 2006 and almost reached a plateau after 2009 (23). A recent review of DA-associated ICDs reported to the FDA indicated that $62 \%$ of the patients experiencing ICDs were treated for PD, $24 \%$ for RLS and 3.5\% for hyperprolactinemia (24). The significantly higher number of reports in patients receiving DA therapy for PD and RLS support the involvement of mesolimbic D3 receptors; pramipexole and ropironole have higher D3 affinity than cabergoline and bromocriptine. In patients with PD who were treated with DAs, there is a reported prevalence of ICDs between 2.6 and $34.8 \%$ (4). Higher rates were noted in specific populations: $39.1 \%$ in patients treated for at least 3 consecutive months (25) and 58.3\% in early-onset PD (26). In patients treated for RLS, ICDs prevalence was lower, at $7.1-11.4 \%$ (4).

The first case of pathological gambling in a patient treated with DAs for prolactinoma was reported in 2007 in a 47-year-old woman taking a low dose of cabergoline (27). She sought medical attention for major depression and paranoid delusions, having incurred a financial loss of $\$ 700000$. Of note, the patient did not have these problems 25 years previously when she took bromocriptine. Table 1 summarizes the individual case reports of ICD during DA therapy administered for prolactinomas $(24,27,28,29,30,31)$.

Pathologic hypersexuality may include increased libido, sexual thoughts, sexual frequency and high-risk sexual activities. Due to their personal nature, patients may not be forthcoming in reporting these manifestations, which can lead to relationship problems, financial loss, reduced work performance and illicit activities (e.g., engagement in prostitution, promiscuity and theft and use of recreational drugs). The mechanism entails reward pathway stimulation as do other ICDs; also, increasinglevels of testosterone in men taking DA therapy may contribute to the hypersexuality. De Sousa et al. proposed the term 'dopa-testotoxicosis' in their report of eight men with prolactinoma and no prior history of psychiatric disease (32). Patients received cabergoline (0.5-1 mg weekly), bromocriptine (0.625-2.5 mg daily) and/or quinagolide (75 $\mu$ g daily). Testosterone levels were low in six and in the lower half of the normal range in two patients, and increased during DA therapy, with all patients achieving normal testosterone levels. Six patients were bothered by hypersexuality and decreased the dose or stopped the DA, with resolution of symptoms, which did not recur after testosterone replacement or pituitary surgery. Additional case reports in men with prolactinomas who received cabergoline (0.5-2 mg weekly) or bromocriptine (7-15 mg daily) confirmed resolution of hypersexuality after the DA dose decreased or was stopped $(29,33,34,35)$. There are few case reports of hypersexuality in women: three with cabergoline or bromocriptine (33) and one with quinagolide (Table 1) (31). While there are more reports of disruptive hypersexuality in men undergoing DA therapy, testosterone levels were not supraphysiologic and the symptoms resolved after stopping the DA. Hence, the term 'dopa-testotoxicosis' does not seem appropriate.

There are few systematic studies of ICDs in patients treated with DAs for prolactinomas. A cross-sectional study evaluated 20 patients with the Minnesota Impulse Disorder Interview (MIDI). ICDs were identified in two male patients (10\%), both treated with relatively low DA doses. Manifestations included pathological gambling, severe hypersexuality and compulsive eating (34). This study found that ICDs prevalence was comparable to that of the general population, but unfortunately did not include a control group.

Bancos et al. conducted the largest case control study aimed at ICD detection in 77 patients with DA-treated prolactinomas and 70 patients with NFA. Patients were selected through a postal survey sent to 200 patients in each group. The NFA group included more women than the prolactinoma group (64 vs 55\%), with a similar mean age. The overall response rates were not different between men and women, but the responders were older than the nonresponders. Additional phone discussions 
Table 1 Individual case reports of patients with prolactinoma who developed dopamine agonist-associated impulse control disorders (ICDs).

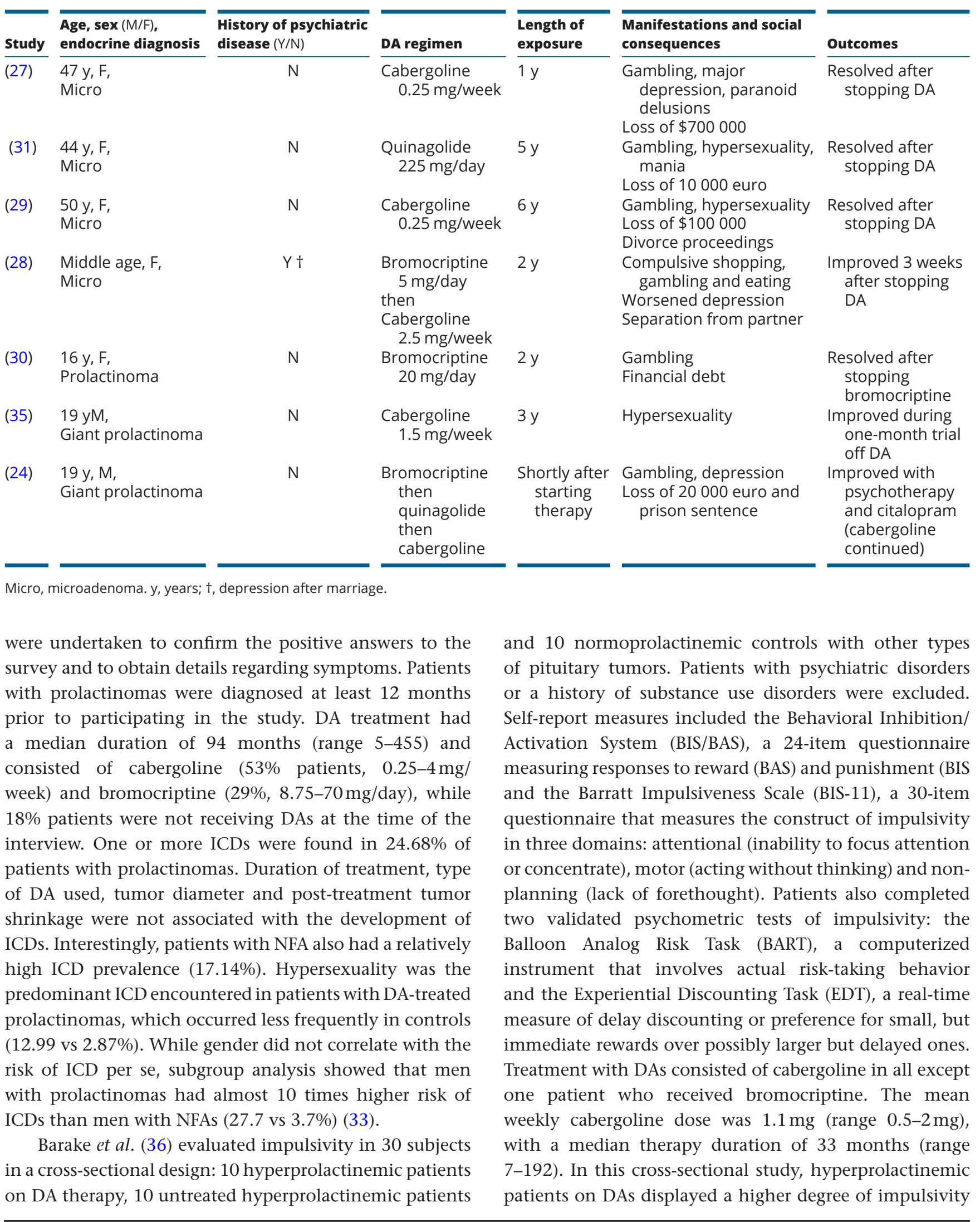


in the attention subscale of the BIS-11 questionnaire as compared to both untreated hyperprolactinemic patients and to normoprolactinemic controls. A higher cabergoline dose was associated with higher impulsive choices on the EDT test in hyperprolactinemic DA-treated group in this study (36).

Celik et al. (22) prospectively evaluated 25 patients with prolactinoma, 31 with NFA and 32 healthy controls for the presence of ICDs and other psychiatric disorders. Two cases of ICDs consisting of hypersexuality (8\%) were associated with DAs use, which improved or resolved after discontinuation of the DA. When patients were exposed to smaller dose of the same DA, either no symptoms or less severe manifestations occurred (22).

The data are sparse regarding risk factors for ICDs in patients with prolactinoma treated with DA therapy. Young age and male gender were associated with ICDs in PD, especially with regard to pathological gambling and hypersexuality (4). However, a correlation between age and ICD development in prolactinoma patients could not be shown. More cases of hypersexuality were reported in men than in women with prolactinoma, but other types of ICDs did not have a gender predilection. Preexisting psychiatric disease, in particular anxiety and depression, may increase the risk of ICD; however, this was an exclusion criterion in some studies. It is unclear whether DA therapy association with ICDs is dose-dependent (suggested by one study that included nine patients taking cabergoline) (36) or related to the duration of DA treatment. Comparing the risk between different DAs is not possible because of different types of DAs that were available at the time of the report in different countries. Challenging patients who experienced ICDs while taking one DA with another DA has been attempted in a few cases and led to reoccurrence of ICD in some patients (24, 28). It is important to note that approximately $33 \%$ of reported patients developed multiple types of ICDs during DA therapy $(32,33)$. Finally, discontinuation of DAs or even reducing the dose along with adding psychotherapy or psychiatric medications were associated with improved or resolved ICDs $(24,27,29,31,32,33,34,35)$.

Based on the data accumulated, we suggest that physicians discuss the risk of ICDs at the time of DA therapy initiation and revisit this issue at follow-up visits. The ICD manifestations reported in the literature were mostly reversible after DA stopped or dose decreased; in some instances, ICDs only developed with one type of DA $(24,27)$. Therefore, in case of mild manifestations, a smaller dose of DA or another DA approved for use in hyperprolactinemia can be attempted, while the patient is carefully monitored by both endocrinology and psychiatry. The antipsychotic aripiprazole has been approved for schizophrenia, bipolar disorder, major depressive disorder and autism spectrum disorder. Unlike most antipsychotics, aripiprazole has partial dopamine agonist D2 activity and has been shown to improve prolactin secretion in some patients with coexistence of a prolactin-secreting tumor and psychiatric disease $(37,38)$. Efficacy and safety studies in patients with prolactinomas are not available. The approach whereby aripiprazole replaces cabergoline or bromocriptine in patients with prolactinoma who developed ICDs requires further study. If the temporal association is demonstrated between pharmacologic treatment of hyperprolactinemia and de novo ICDs, surgical treatment of the pituitary tumor can be considered.

\section{Psychiatric disorders other than ICDs during treatment with dopamine agonists for prolactinoma}

There are reports of DA use associated with severe depression, manic episodes or psychosis, but the overall number is quite small. This is likely an under-reported association, as patients may not be forthcoming in the absence of direct questioning and because of publication bias (clinicians aware of this possibility may not consider it worth presenting). Table 2 summarizes these case reports, with the majority occurring in women taking cabergoline $(37,38,39,40,41,42,43,44,45,46)$. The severity of hyperprolactinemia ranged from 45 to $9400 \mathrm{ng} / \mathrm{mL}$, some patients had prolactinomas and some had a NFA with stalk effect hyperprolactinemia. The psychiatric disturbances included severe depression, mania, psychosis and even catatonia. In most cases, the DA was stopped. In all but one case, an antipsychotic medication was initiated as well. One patient was treated with transsphenoidal resection of the prolactinoma (43). While some patients had a prior history of psychiatric disease, others did not. The duration of exposure to DAs ranged from 4 days to 6 years. In clinical practice, one should always be aware of the potential for the precipitation of psychiatric disease when using DAs, even in the absence of a prior history.

Clinical studies on this topic are sparse. In 1984, Turner et al. (47) performed a retrospective analysis of a 600 patients with acromegaly or hyperprolactinemia treated with DAs. Psychotic reactions were identified in eight patients, among them five women with prolactinoma ages 20-58 treated with bromocriptine (four patients, doses ranging from 7.5 to $60 \mathrm{mg}$ ) or lisuride (one patient, $1.2 \mathrm{mg}$ daily). Psychiatric history prior to DA therapy was negative. Interestingly, 


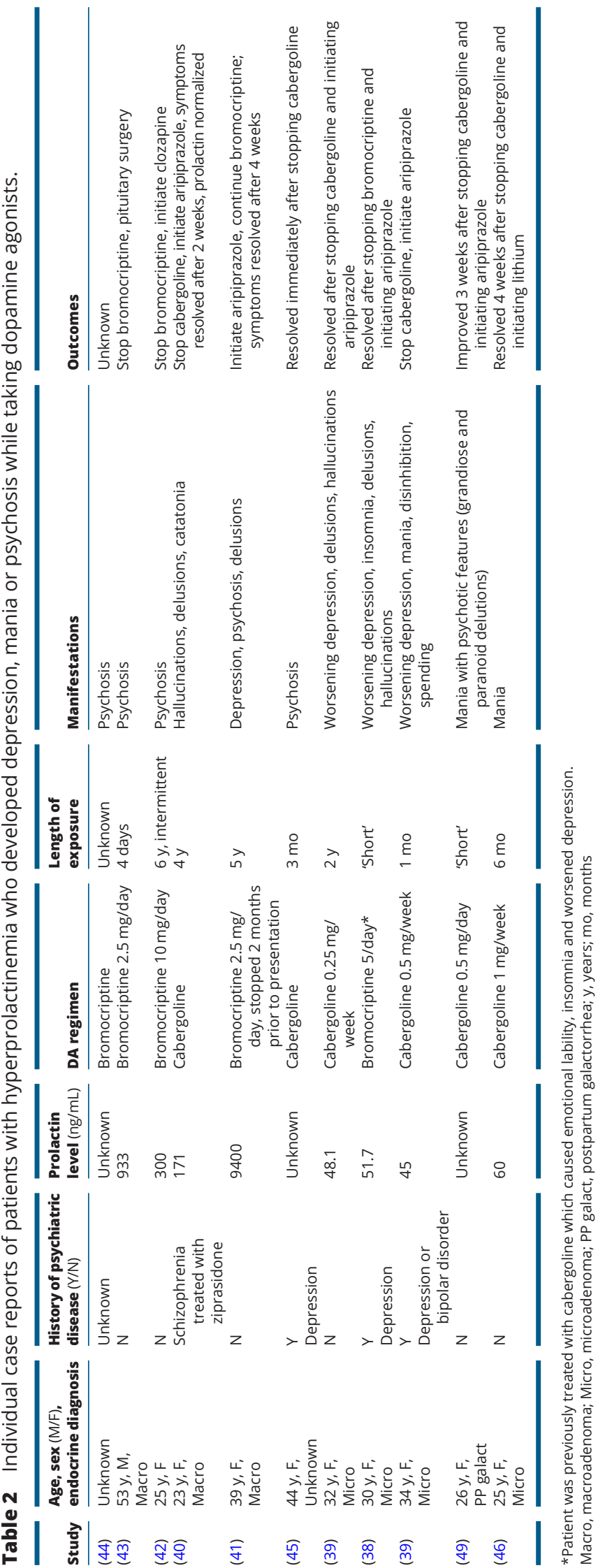

all five patients developed paranoid psychosis. (47). Celic et al. (22) prospectively evaluated 25 patients with prolactinoma treated with cabergoline, 31 with NFA and 32 healthy controls. The study found more patients with prolactinoma screened positive for obsession, interpersonal sensitivity, paranoid ideation after 12 months of DA therapy. The cumulative DA dose was positively correlated to obsession, interpersonal sensitivity, paranoid ideation, hostility, phobic anxiety subscales and higher general severity index scores of SCL-90-R (Symptom Checklist-90-Revised) questionnaire (22).

\section{Treatment of hyperprolactinemia in patients treated with antipsychotic medications}

Particularly challenging is the treatment of hyperprolactinemia in the setting of known psychotic disorders, as the psychiatric medications of choice in this setting are often dopamine antagonists which may further raise prolactin (7). Risperidone and haloperidol are the most commonly reported psychotropic medications that cause hyperprolactinemia. The differential diagnosis of medication-induced hyperprolactinemia vs pituitary adenoma should be carefully considered. Current recommendations suggest withdrawal of any potentially offending drug for 3 days and repeating serum prolactin levels, which must be done in consultation with the patient's mental health specialist (1). If this approach does not seem safe with regard to the patient's psychiatric condition, an MRI of the pituitary gland should be considered. In patients with drug-induced hyperprolactinemia, evaluation for galactorrhea and hypogonadism is necessary. Whenever possible, alternative psychiatric medications that do not raise serum prolactin levels can be considered in collaboration with the patient's psychiatrist. In asymptomatic patients without hypogonadism (or taking gonadal replacement) the prolactin levels can be monitored. Similarly, small pituitary tumors can be closely observed. The antipsychotic aripiprazole may possess unique advantages in this specific setting (48). Unlike other antipsychotics, aripiprazole has partial dopamine agonist activity. Table 2 illustrates that aripiprazole was a satisfactory treatment in many of the reported cases $(37,38,39,40,41,49)$. Prolactin decreased on average by $74 \%$ in patients taking aripiprazole (50) and in many cases normalized. The combination of aripiprazole and bromocriptine or quinagolide was successful in patients who had both macroprolactinomas and schizophrenia $(41,51)$; while these results are encouraging, it is not known 
whether the same degree of tumor involution would be expected. Finally, surgical therapy for prolactinoma was used in some cases in the setting of mass effect manifestations coexistent psychiatric disease $(52,53)$.

\section{Advice for clinical practice}

The prevalence of psychiatric complications from DAs used for hyperprolactinemia and the risk profile of affected patients are not well defined. Counseling all patients is worthwhile given the significant health and societal consequences that may occur during DA therapy. Before prescribing DA, it would be important to take a thorough history of psychiatric disease, and to initiate communication with psychiatry when necessary. Until further research identifies the standardized questionnaires best suited for patients with prolactinomas, the physicians may use direct questioning regarding prior or current history of ICDs, depression, anxiety or psychiatric medications. At follow-up visits, direct questions about changes in mood and behavioral changes are encouraged. If such alterations were identified, the patients would benefit from a multidisciplinary care that includes neuropsychology and psychiatry. Further prospective studies are necessary to make specific clinical recommendations regarding management of psychiatric complications of DA therapy.

\section{Conclusions}

Psychological changes in patients treated with DAs for hyperprolactinemia and prolactinomas vary in spectrum and severity and may have significant medical and social consequences. Physicians who prescribe DAs should be aware of the potential occurrence of ICDs, depression, mania and other types of psychosis. For patients with known psychiatric disease, a close collaboration with mental health specialists is required to assess individualized treatment for prolactinomas and the safety of adding DA therapy.

\section{Declaration of interest}

The authors declare that there is no conflict of interest that could be perceived as prejudicing the impartiality of this study.

\section{Funding}

This research did not receive any specific grant from any funding agency in the public, commercial or not-for-profit sector.

\section{References}

1 Melmed S, Casanueva FF, Hoffman AR, Kleinberg DL, Montori VM, Schlechte JA, Wass JA \& Endocrine S. Diagnosis and treatment of hyperprolactinemia: an Endocrine Society clinical practice guideline. Journal of Clinical Endocrinology and Metabolism 201196 273-288. (https://doi.org/10.1210/jc.2010-1692)

2 Chanson P \& Maiter D. Prolactinoma. In Pituitary, edn 4, ch 16, pp 467-514. Ed S Melmed. Elsevier, 2017. (https://doi.org/10.1016/ B978-0-12-804169-7.00016-7)

3 Molitch ME. Diagnosis and treatment of pituitary adenomas: a review. JAMA 2017317 516-524. (https://doi.org/10.1001/jama.2016.19699)

4 Grall-Bronnec M, Victorri-Vigneau C, Donnio Y, Leboucher J, Rousselet M, Thiabaud E, Zreika N, Derkinderen P \& Challet-Bouju G. Dopamine agonists and impulse control disorders: a complex association. Drug Safety 201841 19-75. (https://doi.org/10.1007/ s40264-017-0590-6)

5 Ben-Jonathan N \& Hnasko R. Dopamine as a prolactin (PRL) inhibitor. Endocrine Reviews 200122 724-763. (https://doi. org/10.1210/edrv.22.6.0451)

6 Ghahremani DG, Lee B, Robertson CL, Tabibnia G, Morgan AT, De Shetler N, Brown AK, Monterosso JR, Aron AR, Mandelkern MA et al. Striatal dopamine $\mathrm{D}(2) / \mathrm{D}(3)$ receptors mediate response inhibition and related activity in frontostriatal neural circuitry in humans. Journal of Neuroscience 201232 7316-7324. (https://doi.org/10.1523/ JNEUROSCI.4284-11.2012)

7 Noronha S, Stokes V, Karavitaki N \& Grossman A. Treating prolactinomas with dopamine agonists: always worth the gamble? Endocrine 201651 205-210. (https://doi.org/10.1007/s12020-0150727-2)

8 Barlier A \& Jaquet P. Quinagolide - a valuable treatment option for hyperprolactinaemia. European Journal of Endocrinology 2006154 187-195. (https://doi.org/10.1530/eje.1.02075)

9 Ahlskog JE. Pathological behaviors provoked by dopamine agonist therapy of Parkinson's disease. Physiology and Behavior 2011104 168-172. (https://doi.org/10.1016/j.physbeh.2011.04.055)

10 Seeman P. Parkinson's disease treatment may cause impulse-control disorder via dopamine D3 receptors. Synapse 201569 183-189. (https://doi.org/10.1002/syn.21805)

11 Athanasoulia AP, Sievers C, Ising M, Brockhaus AC, Yassouridis A, Stalla GK \& Uhr M. Polymorphisms of the drug transporter gene ABCB1 predict side effects of treatment with cabergoline in patients with PRL adenomas. European Journal of Endocrinology 2012167 327-335. (https://doi.org/10.1530/EJE-12-0198)

12 Sobrinho LG. Prolactin, psychological stress and environment in humans: adaptation and maladaptation. Pituitary 20036 35-39. (https://doi.org/10.1023/A:1026229810876)

13 Fava GA, Fava M, Kellner R, Serafini E \& Mastrogiacomo I. Depression hostility and anxiety in hyperprolactinemic amenorrhea. Psychotherapy and Psychosomatics 198136 122-128. (https://doi. org/10.1159/000287535)

14 Kellner R, Buckman MT, Fava GA \& Pathak D. Hyperprolactinemia, distress, and hostility. American Journal of Psychiatry 1984141 759-763. (https://doi.org/10.1176/ajp.141.6.759)

15 Zacur HA, Chapanis NP, Lake CR, Ziegler M \& Tyson JE. Galactorrhea-amenorrhea: psychological interaction with neuroendocrine function. American Journal of Obstetrics and Gynecology 1976125 859-862. (https://doi.org/10.1016/00029378(76)90863-2)

16 Buckman MT \& Kellner R. Reduction of distress in hyperprolactinemia with bromocriptine. American Journal of Psychiatry 1985142 242-244. (https://doi.org/10.1176/ ajp.142.2.242)

17 Fava M, Fava GA, Kellner R, Buckman MT, Lisansky J, Serafini E, DeBesi L \& Mastrogiacomo I. Psychosomatic aspects of 
hyperprolactinemia. Psychotherapy and Psychosomatics 198340 257-262. (https://doi.org/10.1159/000287773)

18 Rocco A, Mori F, Baldelli R, Aversa A, Munizzi MR, Nardone MR, Fabbrini A \& Falaschi P. Effect of chronic bromocriptine treatment on psychological profile of patients with PRL-secreting pituitary adenomas. Psychoneuroendocrinology 199318 57-66. (https://doi. org/10.1016/0306-4530(93)90055-P)

19 Johnson MD, Woodburn CJ \& Vance ML. Quality of life in patients with a pituitary adenoma. Pituitary 20036 81-87. (https://doi. org/10.1023/B:PITU.0000004798.27230.ed)

20 Kars M, van der Klaauw AA, Onstein CS, Pereira AM \& Romijn JA. Quality of life is decreased in female patients treated for microprolactinoma. European Journal of Endocrinology 2007157 133-139. (https://doi.org/10.1530/EJE-07-0259)

21 Athanasoulia AP, Ising M, Pfister H, Mantzoros CS, Stalla GK $\&$ Sievers C. Distinct dopaminergic personality patterns in patients with prolactinomas: a comparison with nonfunctioning pituitary adenoma patients and age- and gender-matched controls. Neuroendocrinology 201296 204-211. (https://doi. org/10.1159/000335996)

22 Celik E, Ozkaya HM, Poyraz BC, Saglam T \& Kadioglu P. Impulse control disorders in patients with prolactinoma receiving dopamine agonist therapy: a prospective study with 1 year follow-up. Endocrine 2018 Epub.

23 Moore TJ, Glenmullen J \& Mattison DR. Reports of pathological gambling, hypersexuality, and compulsive shopping associated with dopamine receptor agonist drugs. JAMA Internal Medicine 2014174 1930-1933.

24 Athanasoulia-Kaspar AP, Popp KH \& Stalla GK. Neuropsychiatric and metabolic aspects of dopaminergic therapy: perspectives from an endocrinologist and a psychiatrist. Endocrine Connections 20187 R88-R94. (https://doi.org/10.1530/EC-18-0030)

25 Bastiaens J, Dorfman BJ, Christos PJ \& Nirenberg MJ. Prospective cohort study of impulse control disorders in Parkinson's disease. Movement Disorders 201328 327-333. (https://doi.org/10.1002/ mds.25291)

26 van Holst RJ, van den Brink W, Veltman DJ \& Goudriaan AE. Why gamblers fail to win: a review of cognitive and neuroimaging findings in pathological gambling. Neuroscience and Biobehavioral Reviews 2010 34 87-107. (https://doi.org/10.1016/j.neubiorev.2009.07.007)

27 Davie M. Pathological gambling associated with cabergoline therapy in a patient with a pituitary prolactinoma. Journal of Neuropsychiatry and Clinical Neurosciences 200719 473-474. (https://doi.org/10.1176/ jnp.2007.19.4.473)

28 Almanzar S, Zapata-Vega MI \& Raya JA. Dopamine agonistinduced impulse control disorders in a patient with prolactinoma. Psychosomatics 201354 387-391. (https://doi.org/10.1016/j. psym.2012.10.002)

29 Falhammar H \& Yarker JY. Pathological gambling and hypersexuality in cabergoline-treated prolactinoma. Medical Journal of Australia 2009 19097.

30 Thondam SK, Alusi S, O'Driscoll K, Gilkes CE, Cuthbertson DJ $\&$ Daousi C. Impulse control disorder in a patient on long-term treatment with bromocriptine for a macroprolactinoma. Clinical Neuropharmacology 201336 170-172. (https://doi.org/10.1097/ WNF.0b013e31829fc165)

31 Vinkers DJ \& van der Wee NJ. A case of mania after long-term use of quinagolide. General Hospital Psychiatry 200729 464. (https://doi. org/10.1016/j.genhosppsych.2007.05.004)

32 De Sousa SM, Chapman IM, Falhammar H \& Torpy DJ. Dopatestotoxicosis: disruptive hypersexuality in hypogonadal men with prolactinomas treated with dopamine agonists. Endocrine $2017 \mathbf{5 5}$ 618-624. (https://doi.org/10.1007/s12020-016-1088-1)

33 Bancos I, Nannenga MR, Bostwick JM, Silber MH, Erickson D \& Nippoldt TB. Impulse control disorders in patients with dopamine agonist-treated prolactinomas and nonfunctioning pituitary adenomas: a case-control study. Clinical Endocrinology 201480 863-868. (https://doi.org/10.1111/cen.12375)

34 Martinkova J, Trejbalova L, Sasikova M, Benetin J \& Valkovic P. Impulse control disorders associated with dopaminergic medication in patients with pituitary adenomas. Clinical Neuropharmacology 2011 34 179-181. (https://doi.org/10.1097/WNF.0b013e3182281b2f)

35 Bulwer C, Conn R, Shankar A, Ferrau F, Kapur S, Ederies A, Korbonits M \& Spoudeas HA. Cabergoline-related impulse control disorder in an adolescent with a giant prolactinoma. Clinical Endocrinology 201786 862-864. (https://doi.org/10.1111/cen.13339)

36 Barake M, Evins AE, Stoeckel L, Pachas GN, Nachtigall LB, Miller KK, Biller BM, Tritos NA \& Klibanski A. Investigation of impulsivity in patients on dopamine agonist therapy for hyperprolactinemia: a pilot study. Pituitary 201417 150-156. (https://doi.org/10.1007/ s11102-013-0480-6)

37 Burback L. Management of a microprolactinoma with aripiprazole in a woman with cabergoline-induced mania. Endocrinology, Diabetes and Metabolism Case Reports 20152015150100.

38 Bakker IC, Schubart CD \& Zelissen PM. Successful treatment of a prolactinoma with the antipsychotic drug aripiprazole. Endocrinology, Diabetes and Metabolism Case Reports 20162016160028.

39 Rovera C, Cremaschi L, Thanju A, Fiorentini A, Mauri MC, Serati M, Lindenmayer JP \& Altamura AC. Cabergoline can induce mania with psychotic features in bipolar I disorder: a case report. Asian Journal of Psychiatry 201622 94-95. (https://doi.org/10.1016/j.ajp.2016.05.010)

40 Freeman B, Levy W \& Gorman JM. Successful monotherapy treatment with aripiprazole in a patient with schizophrenia and prolactinoma. Journal of Psychiatric Practice 200713 120-124. (https://doi.org/10.1097/01.pra.0000265771.47153.a0)

41 Sheldrick AJ \& Grunder G. Aripiprazole reduces serum prolactin in a woman with prolactinoma and acute psychosis. Pharmacopsychiatry 200841 160. (https://doi.org/10.1055/s-2008-1076721)

42 Al-Semaan YM, Clay HA \& Meltzer HY. Clozapine in treatment of bromocriptine-induced psychosis. Journal of Clinical Psychopharmacology 199717 126-128. (https://doi. org/10.1097/00004714-199704000-00016)

43 Peter SA, Autz A \& Jean-Simon ML. Bromocriptine-induced schizophrenia. Journal of the National Medical Association 199385 700-701.

44 Cabeza GA, Flores LF, Iniguez IE, Calarco ZE \& Valencia PF. Acute psychosis secondary to bromocriptine treatment in a patient with a prolactinoma. Revista de Investigación Clínica 198436 147-149.

45 Bilal L \& Ching C. Cabergoline-induced psychosis in a patient with undiagnosed depression. Journal of Neuropsychiatry and Clinical Neurosciences 201224 E54. (https://doi.org/10.1176/appi. neuropsych.11110348)

46 Mohapatra S \& Nayak MR. Cabergoline-induced mania in a patient of pituitary microadenoma. Indian Journal of Psychological Medicine 201739 350-351. (https://doi.org/10.4103/0253-7176.207314)

47 Turner TH, Cookson JC, Wass JA, Drury PL, Price PA \& Besser GM. Psychotic reactions during treatment of pituitary tumours with dopamine agonists. BMJ 1984289 1101-1103. (https://doi. org/10.1136/bmj.289.6452.1101)

48 Ali S, Miller KK \& Freudenreich O. Management of psychosis associated with a prolactinoma: case report and review of the literature. Psychosomatics 201051 370-376. (https://doi.org/10.1016/ S0033-3182(10)70718-0)

49 Yuksel RN, Elyas Kaya Z, Dilbaz N \& Cingi Yirun M. Cabergoline-induced manic episode: case report. Therapeutic Advances in Psychopharmacology 20166 229-231. (https://doi. org/10.1177/2045125315626345)

50 Hoffer ZS, Roth RL \& Mathews M. Evidence for the partial dopaminereceptor agonist aripiprazole as a first-line treatment of psychosis in patients with iatrogenic or tumorogenic hyperprolactinemia. Psychosomatics 200950 317-324. (https://doi.org/10.1176/appi. psy.50.4.317) 
51 Broekhof R, Gosselink MJ, Pijl H \& Giltay EJ. The effect of aripiprazole and quinagolide, a dopamine agonist, in a patient with symptomatic pituitary prolactinoma and chronic psychosis. General Hospital Psychiatry 201234 209.e1-209.e3.

52 Santos Andrade EH, Pan PM, da Silva PF \& Gadelha A. New insights in the management of antipsychotics in the treatment of schizophrenia in a patient with prolactinoma: a case report and review of the literature. Case Reports in Medicine 20102010573252. 53 Liu W, Zahr RS, McCartney S, Cetas JS, Dogan A \& Fleseriu M. Clinical outcomes in male patients with lactotroph adenomas who required pituitary surgery: a retrospective single center study. Pituitary 2018 Epub.

Received 15 August 2018

Revised version received 5 October 2018

Accepted 25 October 2018 